\title{
WEAK $A$-CONVEX ALGEBRAS
}

\author{
ALLAN C. COCHRAN
}

\begin{abstract}
Necessary and sufficient conditions are given in terms of $E^{\prime}$ that a weak topology $w\left(E, E^{\prime}\right)$ on an algebra $E$ be $A$ convex. The main condition is that each element $g$ of $E^{\prime}$ contain a weakly closed subspace $L$ of finite codimension such that $g$ is bounded on all multiplicative translates of $L$. For weak topologies, $A$-convexity (which assumes only separate continuity of multiplication) is equivalent to joint continuity of multiplication.
\end{abstract}

Let $E$ be an algebra, $E^{\prime}$ a total subspace of the dual of $E$ and $w\left(E, E^{\prime}\right)$ the weak topology of $E$ determined by $E^{\prime}$. The purpose of this paper is to determine necessary and sufficient conditions that $w\left(E, E^{\prime}\right)$ be $A$-convex. Warner [4] has given a necessary and sufficient condition that $w\left(E, E^{\prime}\right)$ be locally $m$-convex and also a necessary and sufficient condition that multiplication be jointly $\left(w\left(E, E^{\prime}\right)\right)$ continuous. One of the equivalent forms of our condition is that $w\left(E, E^{\prime}\right)$ is $A$-convex (which requires only the separate continuity of multiplication) if and only if multiplication is jointly $\left(w\left(E, E^{\prime}\right)\right)$ continuous. Thus, all weak topological algebras (joint continuity of multiplication) are already $A$-convex. $A$-convex algebras, which include the locally $m$-convex algebras, were introduced in [2]. In $\$ 2$ the basic properties are given along with some examples. The main results are given in $\$ 3$.

2. A-convex algebras. Throughout this note, $E$ will denote an algebra, $E^{\prime}$ a total subspace of the dual of $E$ and $w\left(E, E^{\prime}\right)$ the weak topology on $E$ induced by $E^{\prime}$. The proofs of the results given here may be found in [2].

(2.1) Definition. A subset $V$ of $E$ is called $A$-convex if $V$ is absolutely convex, absorbing and for each $x \in E, V$ absorbs $x V$ and $V x$.

The inverse image of an $A$-convex set under a homomorphism is $A$-convex, as is the image of an $A$-convex set under a surjective homomorphism.

(2.2) Definition. An $A$-convex algebra is an algebra $E$ together with a topology on $E$ whose neighborhood system at zero has a basis of $A$-convex sets. 1969.

Presented to the Society, January 23, 1970; received by the editors October 3,

AMS 1968 subject classifications. Primary 4650; Secondary 4601, 4625, 1620.

Key words and phrases. $A$-convex algebra, locally $m$-convex algebra, weak topology, topological algebra. 
(2.3) Definition. A seminorm $p$ on $E$ is called $m$-absorbing if for all $x$ in $E$ there are constants $M_{x}$ and $N_{x}$ with

(i) $p(x y) \leqq M_{x} p(y)$, for all $y$ in $E$; and

(ii) $p(y x) \leqq N_{x} p(y)$, for all $y$ in $E$.

Thus, an $A$-convex algebra is an algebra $E$ with a topology determined by a family of $m$-absorbing seminorms. $A$-convexity is preserved with respect to taking subspaces, products and quotients. Each $A$-convex algebra can be topologically and algebraically embedded in an $A$-convex algebra with identity. It is clear from the definition that multiplication is separately continuous.

(2.4) Example. Any locally $m$-convex (hence also Banach) algebra is $A$-convex.

(2.5) ExAmple. Let $C[0,1]$ denote the algebra of continuous realvalued functions on $[0,1]$ (with pointwise operations). A norm on $C[0,1]$ is given by

$$
p(f)=\sup \{|f(x) \phi(x)|: x \in[0,1]\},
$$

where $\phi(x)=x, 0 \leqq x \leqq \frac{1}{2}$ and $\phi(x)=1-x, \frac{1}{2}<x \leqq 1 .(C[0,1], p)$ is a normed linear space which is $A$-convex (not locally $m$-convex). An $A$-convex algebra which is normable is called an $A$-normed algebra. The space $(C[0,1], p)$ is not complete.

(2.6) Example. Let $C_{b}(R)$ denote the algebra of bounded continuous complex-valued functions on $R$ (pointwise operations). Let $C_{0}^{+}(R)$ denote the set of strictly positive real-valued continuous functions on $R$ which vanish at infinity. For each $\phi \in C_{0}^{+}(R)$, let

$$
p_{\phi}(f)=\sup \{|f(x) \phi(x)|: x \in R\}, \quad f \in C_{b}(R) .
$$

Then $p_{\phi}$ is a seminorm and the topology $\beta$ determined by $\left\{p_{\phi}: \phi \in C_{0}^{+}(R)\right\}$ is an $A$-convex topology on $C_{b}(R)$. This so-called weighted space is a complete $A$-convex algebra with identity which is not locally $m$-convex (see [2], [6]).

(2.7) Theorem. An algebra $E$ with a locally convex linear topology for which multiplication is separately continuous is $A$-convex if and only if it is isomorphic to a subalgebra of a product of $A$-normed algebras.

The relationship between $A$-convex and locally $m$-convex algebras is given by the fact that a barrelled $A$-convex algebra is locally $m$-convex.

For the remainder of this paper the following notations will be used: For a linear functional $g$ on $E, K(g)$ will denote the kernel of $g$. The polar of a set $V$ in $E^{\prime}$, taken in $E$, will be denoted by $V^{\circ}$. 
3. Weak $A$-convex algebras. In the proof of the main two theorems the following results will be needed. The proof of Lemma 3.1 may be found in [4] and the proof of Lemma 3.2 is omitted.

(3.1) Lemma. Let $V$ be a w(E, $\left.E^{\prime}\right)$-neighborhood of zero. Then $L=\bigcap\left\{K(v): v \in V^{0}\right\}$ is a $w\left(E, E^{\prime}\right)$-closed subspace of finite codimension.

(3.2) Lemma. Let $g$ be a linear functional on $E$ and $L$ a subspace of $K(g)$. Then $E L \subseteq K(g)$ is equivalent to the property

$\left(^{*}\right)$ for all $x$ in $E$ there is a constant $M_{x}$ such that $|g(x L)| \leqq M_{x}$. Also, $L E \subseteq K(g)$ is equivalent to

$\left.{ }^{* *}\right)$ for all $x$ in $E$ there is a constant $N_{x}$ such that $|g(L x)| \leqq N_{x}$.

If $g$ is a linear functional on $E$ and $L$ is a subspace such that $\left(^{*}\right)$ and $\left({ }^{* *}\right)$ hold, then we say that $g$ is bounded on the (multiplicative) translates of $L$.

(3.3) Theorem. Let $E$ be an algebra and $E^{\prime}$ a total subspace of the dual of $E$. Then w(E, $\left.E^{\prime}\right)$ is $A$-convex if and only if for all $g$ in $E^{\prime}, K(g)$ contains a weakly closed subspace $L$ of finite codimension such that $g$ is bounded on all translates of $L$.

Proof. Let $w\left(E, E^{\prime}\right)$ be $A$-convex and $g$ be in $E^{\prime}$. Then $\{g\}^{0}$ contains an $A$-convex weakly closed neighborhood $V$ of zero. Let $L=\bigcap\left\{K(v): v \in V^{0}\right\}$. By Lemma 3.1, $L$ is a weakly closed subspace of finite codimension. Clearly $g$ is in $V^{0}, L \subseteq V^{00}$ and $V=V^{00}$ so $L \subseteq K(g)$.

For $x$ in $E$, the $A$-convexity of $V$ insures the existence of constants $M_{x}$ and $N_{x}$ such that $|g(x L)| \leqq M_{x}$ and $|g(L x)| \leqq N_{x}$. Hence $g$ is bounded on all translates of $L$ and the condition is necessary.

Let $g(\neq 0)$ be in $E^{\prime}$ and $L$ a weakly closed subspace of finite codimension with $L \subseteq K(g)$ and $g$ bounded on all translates of $L$. It suffices to show that $\{g\}^{0}$ contains an $A$-convex weak neighborhood of zero. By the induced map theorem there exists a unique continuous linear functional $\bar{g}$ on $F=E / L$ such that $g=\bar{g} \circ \phi_{1}$, where $\phi_{1}$ denotes the quotient map from $E$ to $F$. Since $F$ is a finite dimensional Hausdorff space, it is normable. We may assume that the norm on $F$ is chosen so that $\bar{g}(\hat{V})=\{z:|z| \leqq 1\}$ where $\hat{V}$ is the unit ball in $F$. The map $\phi_{1}$ is a continuous linear functional so $V=\phi_{1}^{-1}(\hat{V})$ is an absolutely convex absorbing neighborhood of zero. Then

$$
|g(V)|=\left|\bar{g}\left(\phi_{1}(V)\right)\right|=|\bar{g}(\hat{V})| \leqq 1, \quad \text { so } V \subseteq\{g\}^{0} .
$$

We now show that $V$ is $A$-convex: For $x$ in $E, x L \subseteq K(g)$ by Lemma 
3.2 and $[x L]-$ is a closed subspace contained in $K(g)$. By the induced map theorem there is a unique continuous linear functional $g^{*}$ on $H=E /[x L]-$ with $g=g^{*} \circ \phi_{2}$ where $\phi_{2}$ is the quotient map of $E$ to $H$. The map $t_{x}$ of $F$ to $H$ defined by $t_{x}(\hat{y})=[x y] \sim$ is a well-defined linear map from the finite dimensional space $F$ to $H$. Thus, $t_{x}$ is continuous and the image of $F$ is a finite dimensional Hausdorff space. The image of $F$ under $t_{x}$ is normable and the norm may be chosen so that $\left|g^{*}\left(V^{*}\right)\right| \leqq 1$, where $V^{*}$ denotes the unit ball in $H$. It follows from a standard theorem that there exists a constant $M_{x}$ such that

$$
\left\|t_{x}(\hat{y})\right\|_{2} \leqq M_{x}\|\hat{y}\|, \quad \text { for all } \hat{y} \text { in } F,
$$

where \|\|$_{2}$ denotes the norm on $t_{x}(F)$ and \|\| denotes the norm on $F$. If $y$ is in $V, \hat{y}$ is in $\hat{V}$ and $\|[x y] \sim\|_{2} \leqq M_{x}$. Thus,

$$
\left|g^{*}\left(M_{x}^{-1}[x y] \sim\right)\right| \leqq 1 \text { so }\left|g\left(M_{x}^{-1} x y\right)\right| \leqq 1 .
$$

This shows that $x V \subseteq M_{x} V$. Similarly, there is a constant $N_{x}$ with $V x \subseteq N_{x} V$. Hence $V$ is $A$-convex and the condition is sufficient.

The result of Theorem 3.3 gives another interesting consequence: $A$-convexity is equivalent to joint $\left(w\left(E, E^{\prime}\right)\right)$-continuity of multiplication. Hence any weak topological algebra (joint continuity) is an $A$-convex algebra.

(3.4) Theorem. Let $E$ be an algebra. Then w(E, $\left.E^{\prime}\right)$ is A-convex if and only if the multiplication of $E$ is jointly $\left(w\left(E, E^{\prime}\right)\right)$ continuous.

Proof. Theorem 3.3 combined with Lemma 3.2 give the result that $w\left(E, E^{\prime}\right)$ is $A$-convex if and only if for all $g$ in $E^{\prime}, K(g)$ contains a weakly closed subspace $L$ of finite codimension with $E L \subseteq K(g)$ and $L E \subseteq K(g)$. From a theorem of Warner [4, Theorem 2] this is equivalent to the joint continuity of multiplication.

(3.5) CoROllaRy. Let $E$ be a topological algebra with respect to a weak topology. Then $E$ is $A$-convex.

The following problem remains unsolved:

(3.6) Problem. Is there an example of an algebra $E$ and a subspace $E^{\prime}$ of its dual such that $w\left(E, E^{\prime}\right)$ is $A$-convex but not locally $m$-convex?

\section{REFERENCES}

1. R. Arens, $A$ generalization of normed rings, Pacific J. Math. 2 (1952), 455-471. MR 14, 382.

2. A. C. Cochran, C. R. Williams and E. Keown, On a class of topological algebras, Pacific J. Math. (to appear). 
3. E. A. Michael, Locally multiplicatively-convex topological algebras, Mem. Amer. Math. Soc. No. 11 (1952). MR 14, 482.

4. S. Warner, Weak locally multiplicatively-convex algebras, Pacific J. Math. 5 (1955), 1025-1032. MR 17, 876.

5. - Inductive limits of normed algebras, Trans. Amer. Math. Soc. 82 (1956), 190-216. MR 18, 52.

6. W. H. Summers, A representation theorem for biequicontinuous completed tensor products of weighted spaces, Trans. Amer. Math. Soc. 146 (1969), 121-131.

University of Arkansas, Fayetteville, Arkansas 72701 